\title{
Moderno Paramétrico: Para Além da Fenomenologia na Arquitetura
} Parametric Modern: Beyond Phenomenology in Architecture

\author{
Leonardo Luna de Melo Jorge \\ Universidade Federal do Ceará, Brasil \\ leolmjl@hotmail.com \\ Rafael Mourão Fiuza \\ Universidade Federal do Ceará, Brasil \\ rafaelmfiuza@gmail.com
}

\author{
Daniel Cardoso \\ Universidade Federal do Ceará, Brasil \\ danielcardoso@ufc.br
}

\begin{abstract}
Analysis of Mies Van Der Rohe works seeking recurrences and the elements of his formal language aiming to develop a shape grammar. Elaboration of spreadsheet and explanatory images with the parameters and rules that govern these elements and initial sketch of the algorithm in Rhino + Grasshpper.
\end{abstract}

Keywords: Mies Van Der Rohe; Shape Grammar;

\section{Introdução}

O que torna os exemplares da obra de um grande projetista um conjunto coerente, seja no campo da arquitetura, do design ou das artes, é a linguagem. Nela reside a associação do objeto independentemente do seu lugar no mundo ou época a um autor, ou movimento, e o que possibilita essa percepção é o conjunto de atos e escolhas sucessivas de design aos quais todos os objetos foram submetidos antes de tomar sua forma final. Esse processo de pensamento pode ser entendido como uma gramática da forma, que segundo Celani, (2006) é uma estrutura computacional capaz de descrever uma linguagem formal por meio de regras e de um alfabeto.

Mies Van der Rohe foi um dos arquitetos que, com seu conjunto de obras, revolucionou a arquitetura durante 0 período moderno. Porém seu principal legado não reside apenas nas obras materializadas, mas sim na sua forma clara de ver e pensar a arquitetura e o design. Sua linguagem foi expressada em seus edifícios dos mais variados programas e escalas, os quais serviram substrato para o processo analítico. Portanto, o objetivo da pesquisa é agrupar as obras miesianas em um conjunto, declarando linguagem objetiva, composta por regras de composição de elementos característicos, e a partir dessa gramática (regras e elementos) sistematizadas, gerar novos indivíduos virtuais coerentes com sua obra exatamente por se usar da mesma linguagem.

"é nisso que estou trabalhando [uma linguagem comum]. Eu não estou trabalhando em arquitetura, eu estou trabalhando a arquitetura como uma linguagem. Acho que é necessário ter uma gramática para ter uma linguagem. Deve ser uma linguagem viva, mas finalmente é possível conseguir uma gramática." (Mies van der Rohe, 1955)

\section{Metodologia}

Para o exercício analítico de compreensão da gramática formal de Mies, o primeiro passo foi juntar dados sobre a maior quantidade de obras possíveis. As informações coletadas em livros, revistas, e no site do acervo do MOMA com as pranchas originais de seu escritório, foram então agrupadas em uma biblioteca virtual e assim se deu início ao processo de observação. Buscou-se exercitar processo de uma gramática da forma analítica, que segundo Celani, (2006) "a partir de uma sequência dada de palavras, são feitas reduções sucessivas. O resultado é uma variável booleana do tipo "sim" ou "não", que indica se a sequência original pertence ou não à linguagem descrita pela gramática".

Alguns edifícios foram modelados, permitindo encontrar características recorrentes de sua obra e, posteriormente, decompor os organismos edifícios em elementos de composição, os quais puderam ser ferramenta sistemática de leitura de seus projetos. Os elementos consistem em uma série de volumes que, hierarquizados por importância na percepção do objeto, formam o processo linguístico do arquiteto. Cada volume possui suas particularidades formais e visuais, porém sempre pode ser reduzido à um prisma, percebido por princípios da Gestalt como fechamento e continuidade, partindo de seus elementos tridimensionais. $\mathrm{E}$ partindo desses elementos, suas geometrias e posições, criou-se uma planilha na qual para cada edifício, cada volume era descrito com dados numéricos referentes ao seu dimensionamento, e relação de composição tridimensional com os outros volumes. Partindo desses dados foi possível descrever as relações de proporção da lógica compositiva dos volumes.

"A unidade é a cola visual que mantêm tudo coeso. Você sabe que a obteve quando todas as relações visuais no projeto estão organizadas numa relação dependente tão refinada que nela cada elemento sustenta e reforça todos os outros e na qual qualquer mudança, por menor que seja, altera e prejudica a perfeição no equilíbrio e tensão". (Rowena, 1982) 
Com a linguagem compreendida, e o processo generativo de indivíduos esclarecido, transcreveu-se tais informações no software de modelagem paramétrica Grasshopper, a fim de gerar indivíduos e comprovar os resultados obtidos com a obra de Mies Van der Rohe.

\section{Desenvolvimento}

Para descrever de maneira objetiva todos os edifícios catalogados, foram listadas e descritas recorrências, as quais permitiram a identificação dos elementos de composição e seus parâmetros. Partindo da reincidência, selecionaram-se projetos do pós segunda guerra até a morte do arquiteto. São elas:

- Prismas Horizontais: Crown Hall, Neue Nationalgalerie, Biblioteca Martin Luther King, Sede para Bacardi no México, Projeto para Consulado Americano em São Paulo, Casa Farnsworth, Projeto Casa Núcleo / Casa 50x50, Projeto de um Centro de Convenções para Chicago e Correios na Federal Plaza em Chicago;

- Prismas Verticais: Torre Seagram, Torre IBM, Lake Shore Drive $\mathrm{n} \cong 860$ e $\mathrm{n} \cong 880$, Kluczynski Federal Building e Federal Court em Chicago.

\section{Reconhecimento}

A primeira característica percebida indicativa da linguagem miesiana foi a distinção tipológica entre prismas predominantemente horizontais e prismas predominantemente verticais. Tal separação carrega uma série de premissas compositivas, uma vez que cada tipologia é projetada com particularidades.

Posteriormente, observou-se que todas as dimensões do edifício seguiam uma modulação mínima, a qual corresponde à paginação de piso, rebatendo em outros aspectos projetuais, como a estrutura, a divisão dos painéis de fachada, os layouts e o paisagismo.

Na estrutura, as paginações determinam a dimensão dos vãos e a composição desses, ditando ritmos e proporções. Os ritmos, sempre simétricos, constroem visualmente os objetos, e parte da coesão da linguagem se deve a recorrências da quantidade de intercolúnios nos dois eixos.

Esses eixos ordenam a estrutura visual da forma prismática, indicando simetrias e equilíbrios visuais, que podem ser estáticos ou dinâmicos. Entende-se equilíbrio visual estático como algo simétrico e dinâmico como algo que "transmite a sensação" de simétrico, mas não é.

Os elementos ordenados pelos eixos são volumes, que hierarquizados criam a forma do edifício.

\section{Elementos}

Esse conjunto de volumes é composto inicialmente pelo Volume Mestre (VM), a totalidade da forma. Primeiro a ser percebido, delimitado pela estrutura. As relações de proporção, composição e divisão acontecem todas em função dele. Inscritos a esse se encontram os Volumes Contidos (VC), que correspondem à vedação que separa o interior do exterior, ora iguais ao VM, ora recuados dentro desse. Em seguida, os Volumes Nucleares (VN) são dispostos na composição atravessando todos os pavimentos, podendo variar em quantidade e dimensão. Por fim, anexado externamente à maior face do VM, há o Volume Acoplado (VA), correspondendo à um plano horizontal que reforça o equilíbrio visual.
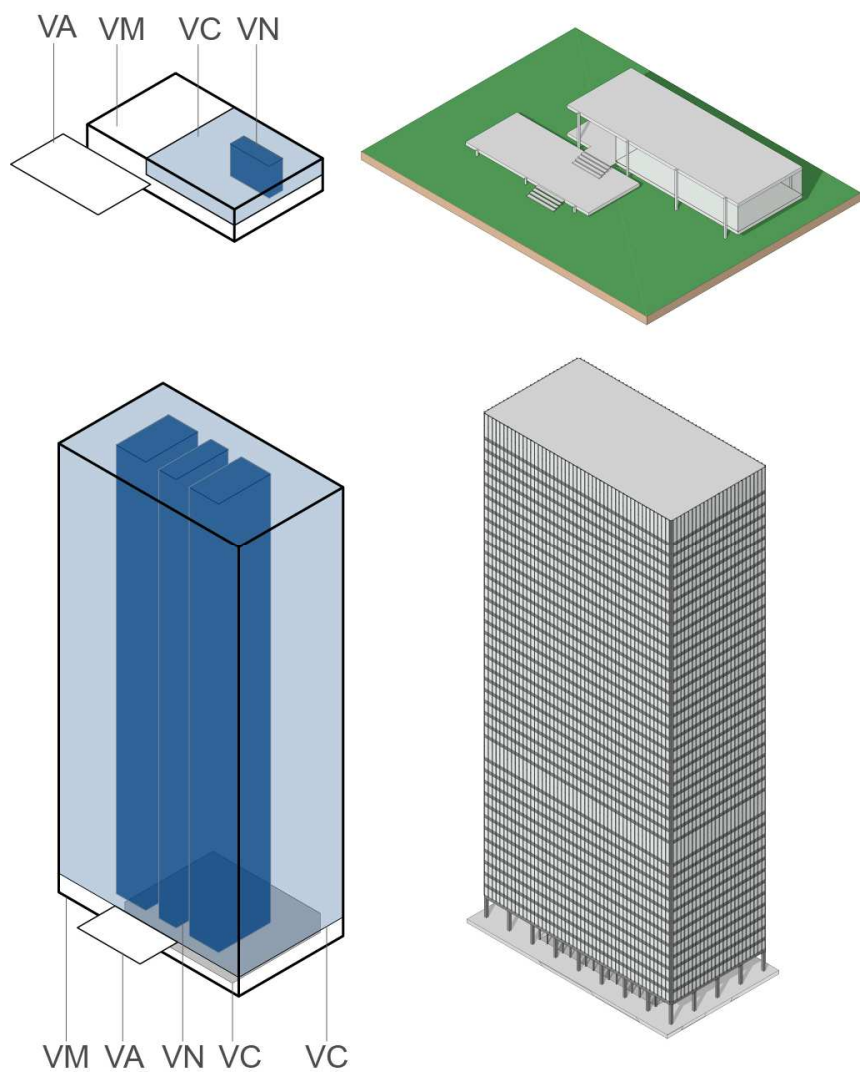

Figura 1: Elementos que compõem a forma.

\section{Parâmetros}

Os parâmetros listados dos volumes foram:

(T) - Tipologia.

$(P x),(P y)$ e $(P z)$ - Dimensão da paginação nos três eixos em centímetros:

(Mx), (My) e (Mz) - Dimensão em quantidade de paginações nos três eixos do VM em número natural;

$(\mathrm{Cx})$, (Cy) e (Cz) - Dimensão em quantidade de paginações nos três eixos do VC em número natural;

$(\mathrm{Nx})$, (Ny) e (Nz) - Dimensão em quantidade de paginações nos três eixos do VN em número natural;

$(A x),(A y)$ e $(A z)$ - Dimensão em quantidade de paginações nos três eixos do VN em número natural;

(Pix) e (Piy) - Quantidade de paginações do Intercolúnio em X e em Y número natural (Apenas no VM);

(Ix) e (ly) - Quantidade de Intercolúnios em X e Y; 
(Piz) - Quantidade de paginações do pavimento em Z em número natural;

(Iz) - Quantidade de Pavimentos em Z;

(Pbx) e (Pby) - Quantidade de paginações do Balanço Visual em $X$ e em $Y$ em número natural (Apenas no $V M$ );

(Fx), (Fy) e (Fz) - Volumes fracionados em X, Y e Z;

(Cex), (Cey) e (Cez) - Excentricidade em paginações do VC nos três eixos em quantidade de paginações;

(Nex) e (Ney) - Excentricidade em paginações do VN em quantidade de paginações;
(Aex), (Aey) e (Aez) - Excentricidade em paginações do VA nos três eixos em quantidade de paginações.

O fracionamento, em todos os eixos, corresponde a divisão do VM nesses eixos, permitindo a locação dos outros volumes em função da compartimentação. Já a excentricidade nada mais é que o deslocamento dos volumes em relação ao centro do VM.

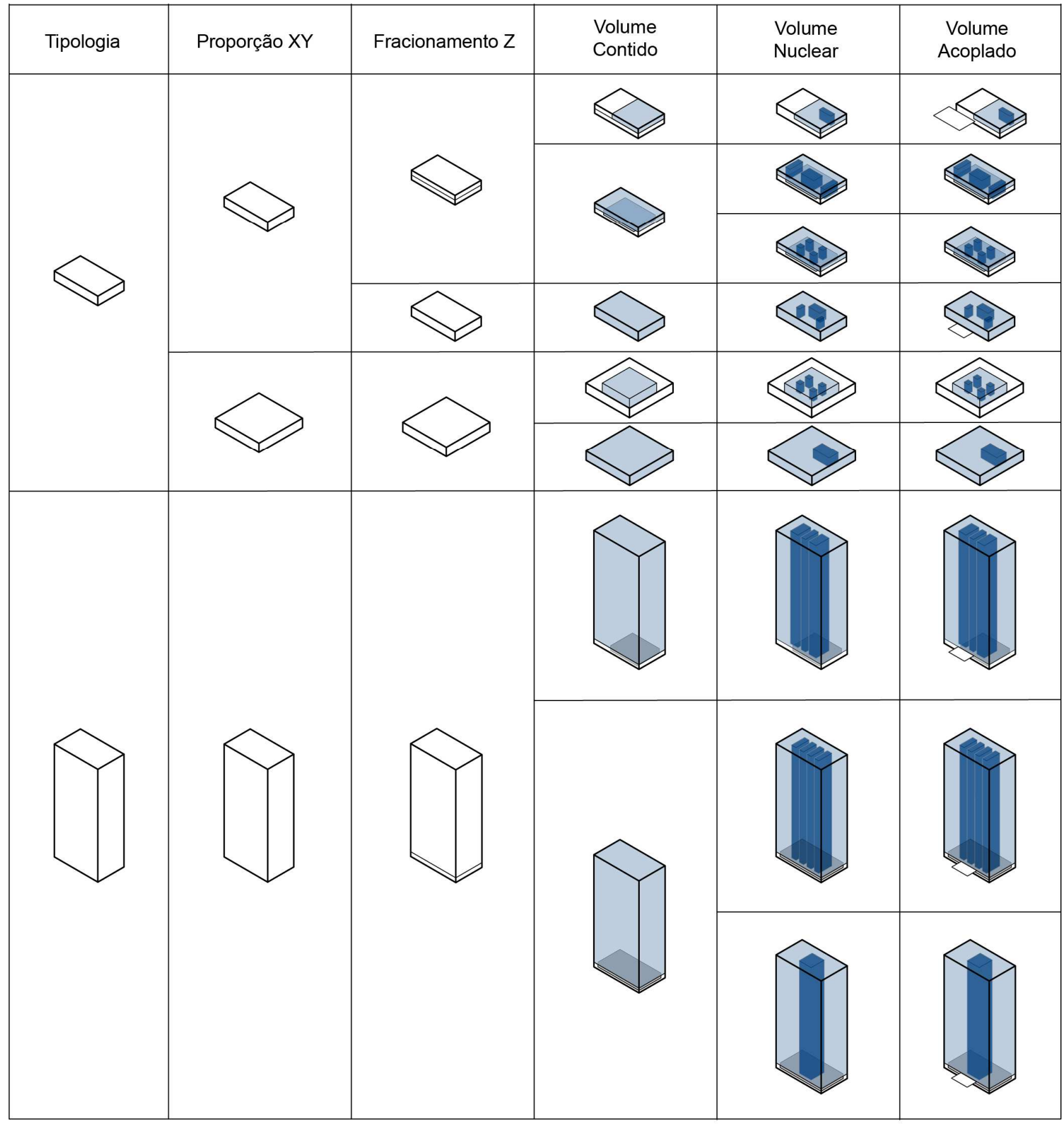

Figura 2: Estados assumidos pelo objeto. 


\section{Construção Lógica}

1. Foram observadas a seguintes regras:

2. Escolhe-se a tipologia formal (T):

a. Prisma Vertical.

b. Prisma Horizontal.

3. Determina-se a dimensões $X(P x), Y(P y)$ e $Z(P z) d a$ paginação.

-Prismas verticais só podem ter paginação $X Y$ quadrada (Proporção 1:1).

-Caso a paginação seja retangular, a proporção da mesma é maior que 1 e menor ou igual a 2.

4. Determina-se a dimensão do vão em função do número de paginações em $X$ (Pix) e $Y$ (Piy).

-A proporção dos intercolúnios está entre 0,5 e 2,1

5. Determina-se o número de intercolúnios nos eixos $X(I x)$ e Y (ly).

-O (Ix) não ultrapassa 13.

-O (ly) não ultrapassa 6.

-Caso seja um prisma vertical o (ly) é 3 ou 4.

6. Determina-se o número de paginações do balanço em $X$ (Pbx) e Y (Pby).

-A proporção entre balanço e intercolúnio no mesmo eixo está entre 0 e 0,5.

-Caso a planta seja quadrada, essa proporção pode chegar 0.625 .

-Os balanços são sempre simétricos num mesmo eixo.

7. Determina-se a altura dos pavimentos em função do número de paginações em Z (Piz).

8. Determina-se o número de pavimentos no eixo $Z$ (Iz).

-Descrição algébrica do
$(\mathrm{Mx})=\left[(\mathrm{Px})^{*}(\mathrm{Ix})^{*}(\mathrm{Pix})\right]+\left[2^{*}(\mathrm{Pbx})^{*}(\mathrm{Px})\right]$
$\left.(\mathrm{My})=[(\mathrm{Py}))^{*}(\mathrm{Iy})^{*}(\mathrm{Piy})\right]+\left[2^{*}(\mathrm{Pby})^{*}(\mathrm{Py})\right]$
$(\mathrm{Mz})=(\mathrm{Pz})^{*}(\mathrm{Iz})^{*}(\mathrm{Piz})$.

9. Determina-se o fracionamento do $\mathrm{VM}$ no eixo $Z(F z)$. Esse fracionamento determina o número de $V C$ 's e suas cotas iniciais e finais, não podendo se cruzar no eixo $Z$.

-Seguem-se os passos 9 e 10 para cada VC.

-Caso haja mais de um fracionamento nos prismas verticais, o $\mathrm{VC}$ de base é o único que pode ter $(C x) \leq(M x)$ e $(C y) \leq(M y)$.

10. Determina-se a quantidade de paginações em $X(C x)$, em Y (Cy) e em Z (Cz) dos VC's.

11. Determina-se as excentricidade em $X$ (Cex), em $Y$ (Cey) VC's.

12. Determina-se o fracionamento do $V M$ no eixo $X(F x)$. -De 1 a 6 fracionamentos.

13. Determina-se o fracionamento no eixo $\mathrm{Y}(\mathrm{Fy})$. $-(\mathrm{Fx})^{\star}(\mathrm{Fy})$ determina o número de VN's . -Para cada VN aplicar passos 13 e 14.

-Caso o fracionamento em $X(F x)$ seja 3 ou mais (Fy) só pode ser 1.

14. Determina-se a quantidade de paginações em $X(N x)$ e em Y (Ny) dos VN's.

-A altura dos VN's (Nz) é igual a (Mz).

15. Determina-se as excentricidades em $X(N e x)$ e em $Y$ (Ney) dos VN's.

16. Determina-se a presença de VA (Va).

a. Sim.

b. Não.
17. Determina-se, caso haja, as dimensões em $X(A x)$ e em $\mathrm{Y}(\mathrm{Ay})$ dos VA's.

-VA's tem alturas $(\mathrm{Az})$ de lajes.

18. Determina-se as excentricidades em $X$ (Aex), em $Y$ (Aey) e em Z (Aez) dos VA's.

-O centro de um VA é externo ao perímetro do VM.

\section{Resultados}

Discriminaram-se as partes do organismo, suas características e suas relações, gerando indivíduos a partir dessa gramática. Iniciou-se a escrita de um algorítmo no Rhino+Grasshopper, locando-se a estrutura de acordo com a forma da planta, sendo plantas quadradas com estrutura nas extremidades e plantas retangulares com estrutura na intersecção de vigas.

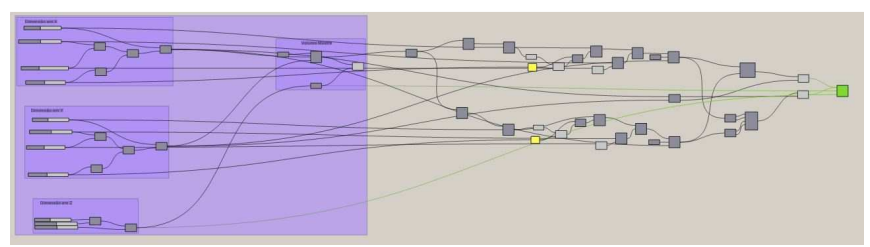

Figura 3: Algoritmo.

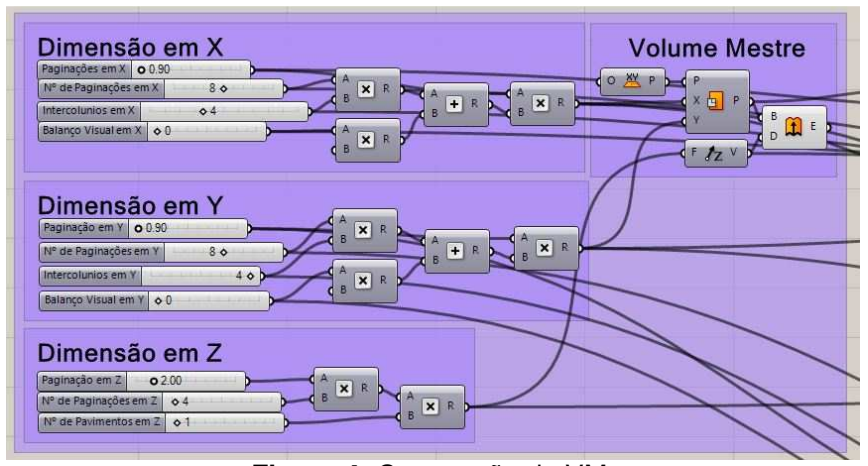

Figura 4: Construção do VM.

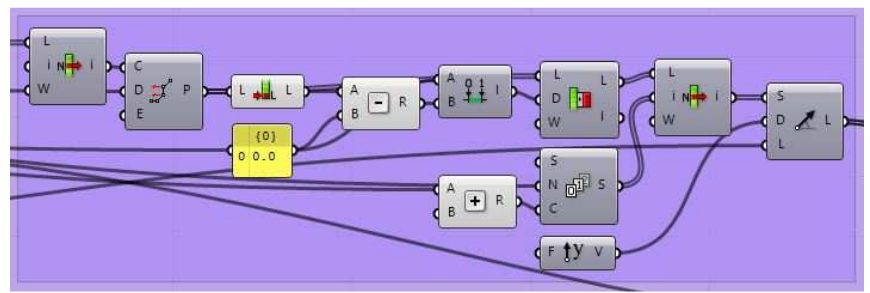

Figura 5: Geração da estrutura em um eixo.

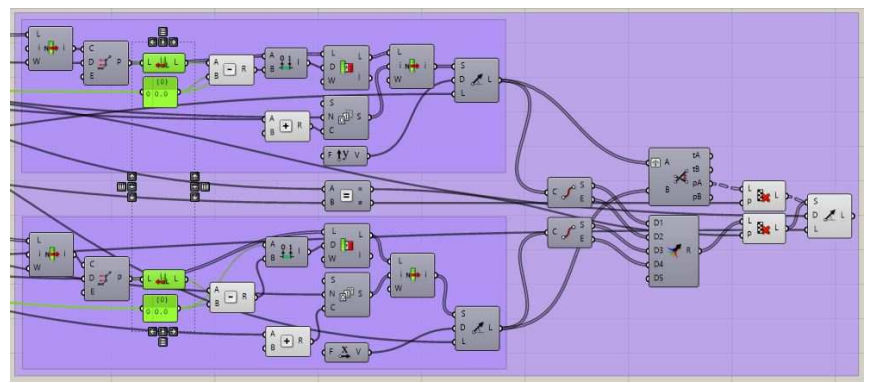

Figura 6: Seletor automático da estrutura. 

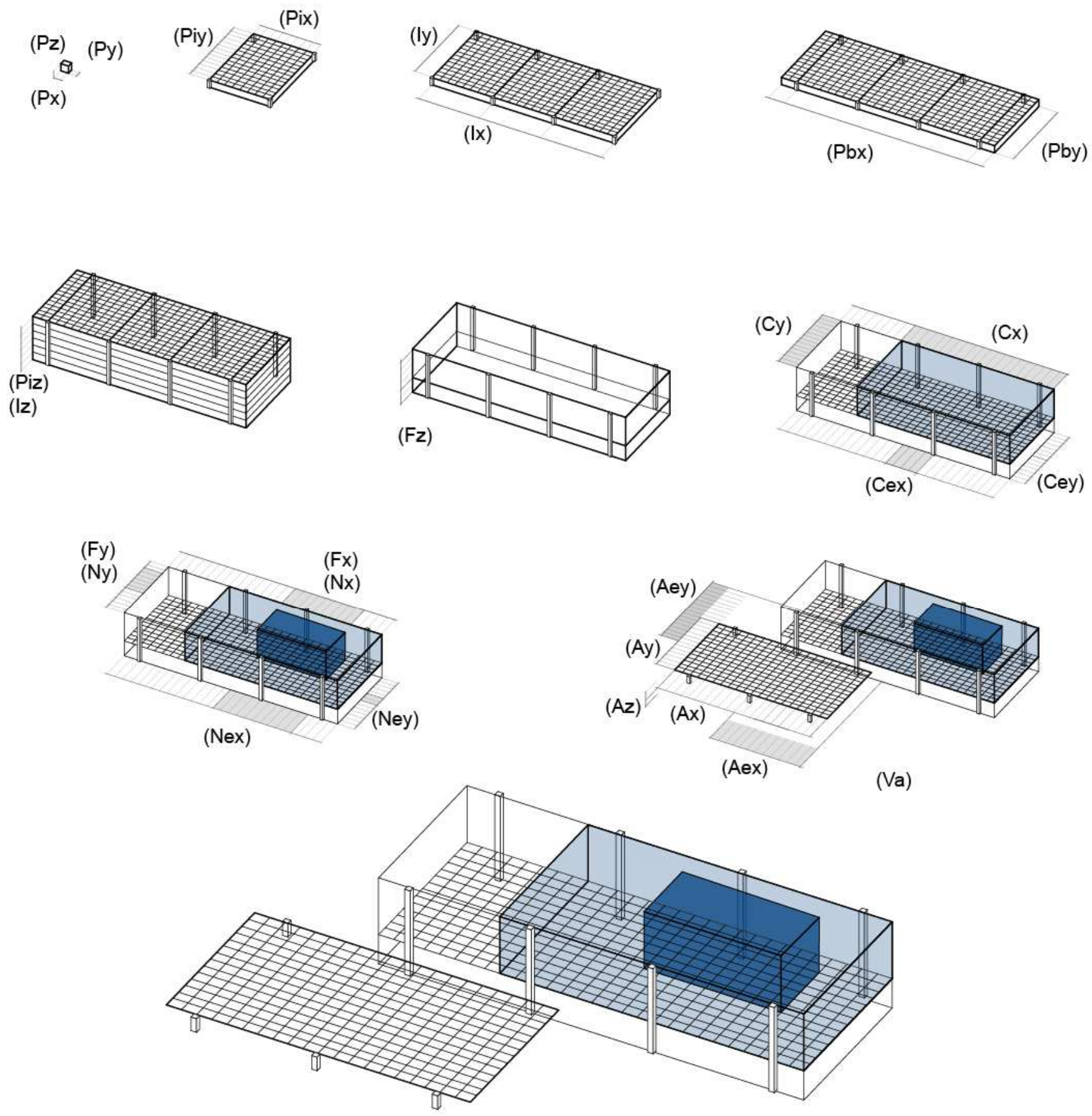

$(P x)=84 \mathrm{~cm} ;(P y)=63 \mathrm{~cm} ;(P z)=84 \mathrm{~cm} ;(P i x)=8 ;(P i y)=14 ;(\mathrm{lx})=3 ;(\mathrm{ly})=1 ;(\mathrm{Pbx})=2 ;(\mathrm{Pby})=0 ;(\mathrm{Piz})=6 ;(\mathrm{Iz})=0 ;(\mathrm{Fz})=2 ;(\mathrm{Cx})=20$; $(C y)=14 ;(C e x)=4 ;(C e y)=0 ;(F x)=1 ;(F y)=1 ;(N x)=9 ;(N y)=6 ;(N e x)=10 ;(N e y)=2 ;(V a)=s i m ;(A x)=20 ;(A y)=14 ;(A z)=1 ;$

$($ Aex $)=12 ;($ Aey $)=16$

Figura 7: Exemplo de aplicação na construção de um objeto com respectivos valores de cada parâmetro. 


\section{Conclusão}

$\mathrm{O}$ arquiteto parte de um prisma e fraciona-o sucessivamente nos três eixos. Essa lógica divisiva independe do programa, uma vez que a lógica compositiva se aplica a todas as obras do recorte. Ela também parece ser análoga a lógica compositiva da implantação, do paisagismo, das fachadas, dos layouts e até o detalhamento e mais pesquisa pode ser feita nesse sentido. Por fim, o equilíbrio visual parece o principal determinante do resultado compositivo, estabelecendo relação entre as excentricidades. Porém não desenvolveu-se um modo de declarar numericamente 0 equilíbrio dinâmico, sendo isso também um objeto de pesquisa.

\section{Referências}

CARDOSO, Daniel. Desenho De Uma Poiesis. 2011. 288 f. Tese (Doutorado) - Curso de Arquitetura, Universidade Federal do Ceará, Fortaleza, 2011.

CELANI, Gabriela et al. A Gramática da Forma como Metodologia de Análise e Síntese em Arquitetura. Conexão: Comunicação e Cultura, Caxias do Sul, v. 5, n. 10, p.180-197, jul/dez. 2006. Semestral.

ZIMMERMAN, Claire; GOSSEL, Peter. Mies Van Der Rohe. Taschen, 2016. 96 p.

MONTANER, Josep Maria. Sistemas Arquitetônicos Contemporâneos. Gustavo Gili, 2010. 224 p.

PUENTE, Moisés (Ed.). Conversas com Mies van der Rohe: Certezas americanas. Gustavo Gili, 2006. 96 p.

HANNAH, Gail Greet. Elementos do Design Tridimensional: Rowena Reed Kostellow e a Estrutura das Relações Visuais. Cosac Naify, 2015. 192 p. Tradução de: Donaldson Garschagen

JOHNSON, Sara. The Proposed Future of Mies van der Rohe's Only Library. 2015.2 Disponível $<$ http://www.architectmagazine.com/design/on-the-boards/theproposed-future-of-mies-van-der-rohes-only-library_0>. Acesso em: 19 jul. 2017.

MOMA. Ludwig Mies van der Rohe. Disponível em: $<$ https://www.moma.org/artists/7166? $=$ undefined\&page $=18 \&$ direc tion=fwd>. Acesso em: 19 jul. 2017.

COLOMBO, Luciana Fornari. O projeto de teatro de Ludwig Mies van der Rohe. 2015. Disponível em: <http://www.vitruvius.com.br/revistas/read/arquitextos/16.185/57 82>. Acesso em: 19 jul. 2017.

GALEAZZI, Ítalo. Mies van der Rohe no Brasil: Projeto para o Consulado dos Estados Unidos em São Paulo, 1957-1962. 2005. Disponível em: <http://www.vitruvius.com.br/revistas/read/arquitextos/05.056/51 1>. Acesso em: 19 jul. 2017. 